\title{
Proporcionalidade corporal na avaliação antropométrica de adolescentes pós-menarca
}

\section{Body proportion in anthropometric assessment of post-menarche adolescents}

Bruna BRONHARA ${ }^{1}$

Valéria Cristina Ribeiro VIEIRA ${ }^{1}$

RE S U M O

\section{Objetivo}

Verificar o comportamento da proporcionalidade corporal em adolescentes pós-menarca e sua influência na avaliação antropométrica desses indivíduos, com ênfase na relação peso/estatura.

\section{Métodos}

Avaliaram-se 80 adolescentes de uma escola pública de Alfenas, M inas Gerais, com pelo menos 12 meses pós-menarca. As medidas antropométricas foram realizadas conforme procedimentos padronizados. A proporcionalidade corporal foi avaliada pelo Índice de Cormic. A população foi dividida em grupos de estudo (Índice de Cormic > mediana) e controle (Índice de Cormic $\leq$ mediana). Os grupos foram comparados em relação às idades cronológica e ginecológica, peso, estatura, estatura tronco-cefálica, comprimento de pernas, índice de massa corporal e indicadores de adiposidade global e localizada.

\section{Resultados}

Os grupos estudados eram homogêneos em relação ao desenvolvimento físico, visto que as médias das idades cronológica e ginecológica não diferiram estatisticamente. A média e o desvio-padrão do Índice de Cormic corresponderam a 0,52 e 0,013, respectivamente. Os grupos não diferiram em relação à estatura $(p=0,23)$, porém o grupos de estudo apresentou valores de estatura tronco-cefálica significantemente superiores em relação ao grupo controle $(p<0,01)$. Proporção significantemente superior $(67,6 \%)$ do grupo de estudo apresentou índice de massa corporal > mediana, em comparação ao grupo controle (37,2\%). Quanto aos indicadores de adiposidade global ou localizada, não foram observadas diferenças estatísticas entre os grupos.

\section{Conclusão}

As diferenças na proporcionalidade corporal não se relacionaram com a estatura. Altos valores de Índice de Cormic parecem estar associados a elevado índice de massa corporal, mas não à composição corporal e à topografia da gordura corporal. Questiona-se, assim, a utilização exclusiva do índice de massa corporal para avaliação antropométrica em serviços de saúde, visto que pode gerar resultados inexatos em populações com comprometimento na proporcionalidade corporal.

Termos de indexação: adolescente; antropometria; composição corporal; estado nutricional.

\footnotetext{
${ }^{1}$ Universidade Federal de Alfenas, Departamento de Nutrição. Rua Gabriel Monteiro da Silva, 714, Centro, 37130-000, Alfenas, MG, Brasil. Correspondência para/Correspondence to: V.C.R. VIEIRA. E-mail: <valnut@unifal-mg.edu.br>.
} 


\section{A B S T R A C T}

\section{Objective}

To verify body proportion performance in pos-menarche adolescents and its influence in anthropometric assessment, emphasizing weight-height ratio.

\section{Methods}

Eighty adolescents from a public school in Alfenas, M inas Gerais, Brazil, were evaluated, with at least 12 months pos-menarche. Anthropometric measurements were obtained according to standardized procedures. Body proportion was assessed by Cormic Index. The population was divided in studied group (Cormic Index > median) and control group (Cormic Index $\leq$ median). The chronological and gynecological ages, weight, height, sitting-height, leg length, body mass index and overall and localized body fat indicators were compared between the groups.

\section{Results}

The means of chronological and gynecological ages did not differ statistically between the groups, showing homogeneity relating to the physical development. The Cormic Index mean and standard deviation corresponded to 0.52 and 0.013 , respectively. The groups did not differ in height $(p=0.23)$, but the studied group showed significantly higher sitting-height values than the control group $(p<0.01)$. A significantly higher proportion $(67.6 \%)$ of the studied group presented body mass index $>$ median, when compared with the control group (37.2\%). The overall and localized body fat indicators were not statistically different between the groups.

\section{Conclusions}

The differences in body proportion were not related to height. Higher Cormic Index values seem to be associated with a higher body mass index, but not with body composition and body fat distribution. Therefore, we question the exclusive use of body mass index in anthropometric assessment in health services, considering that it can generate inexact results in a population with compromised body proportion.

Indexing terms: adolescent; anthropometry; body composition; nutritional status.

\section{N T R O D U Ç Ã O}

Nos países em desenvolvimento, bem como em grupos de baixo nível socioeconômico de países desenvolvidos, os efeitos da desnutrição crônica são, na maioria das vezes, combinados com os de doenças infecciosas ${ }^{1}$. Por ser cumulativo, esse processo de privação, que pode começar dentro do útero e incidir de forma mais intensa no início da infância, pode acarretar comprometimentos não apenas estaturais, mas, também, na proporcionalidade corporal. Tal fato pode determinar que, muitas vezes, essas duas condições estejam associadas².

o índice mais comum utilizado para expressar a proporcionalidade corporal é o chamado Índice de Cormic (IC), obtido pela relação entre a estatura tronco-cefálica (ETC) e a estatura total $(E)(I C=E T C / E)$. É uma medida relativa do comprimento do tronco ou das pernas e varia entre indivíduos e grupos ${ }^{3}$.
Henneberg et al. ${ }^{4}$, em estudo longitudinal com indivíduos de baixa estatura entre 6 e 18 anos de idade, provenientes de níveis socioeconômicos alto e baixo, analisaram as diferenças em relação às dimensões do tronco e membros. Constataram que, apesar de os dois grupos possuírem estaturas idênticas, suas proporções corpóreas diferiam. Em relação aos componentes da estatura, o comprimento do tronco de crianças pobres constituía uma proporção maior em relação ao comprimento total do corpo, em comparação com as crianças de baixa estatura e nível socioeconômico alto.

Vieira ${ }^{5}$, trabalhando com adolescentes pós-menarca de nível socioeconômico baixo, verificou que o grupo de estudo, representado por aquelas com baixa estatura, apresentou IC significantemente maior, em relação às adolescentes com estatura normal.

Norgan \& Jones ${ }^{6}$ também mostraram uma correlação negativa entre estatura e IC, em estudo 
com ingleses adultos, de ambos os sexos. Tal correlação, considerada esperada pelos autores, foi mais forte no sexo feminino.

Por outro lado, a baixa estatura de causa nutricional pode também ser acompanhada pelo aumento do peso para estatura, de forma aparentemente paradoxal ${ }^{1,7,8}$. Segundo Trowbridge et al. ${ }^{1}$, essa situação, diferentemente do que ocorre em nível socioeconômico alto, não poderia ser interpretada como obesidade resultante de ingestão energética acima das necessidades.

Popkin et al. ${ }^{7}$ encontraram, no Brasil, prevalência de $3,5 \%$ de sobrepeso nas crianças de 3 a 6 anos com baixa estatura, enquanto que na África do Sul o percentual foi de 13,1\%, na China de $17,0 \%$ e na Rússia de $48,6 \%$. Apesar da menor prevalência de sobrepeso em crianças de baixa estatura no Brasil, mesmo após o ajuste para a renda, houve associação significante entre baixa estatura e sobrepeso, diferentemente dos outros três países estudados. Os autores questionam o que determinaria esse estado nutricional, aparentemente paradoxal, considerando que as crianças em questão tiveram poucas oportunidades, disponibilidade de recursos e estilos de vida para se tornarem obesas.

Para Post et al. ${ }^{8}$, também é difícil entender como crianças com altos níveis de morbimortalidade, que vivem em péssimas condições socioeconômicas e ambientais e apresentam elevadas prevalências de déficit de estatura para idade, podem ter níveis médios de peso para estatura similares ou mesmo superiores aos de crianças de países desenvolvidos. Para esses autores, a alta relação peso para estatura evidenciaria, na verdade, diferenças na composição e proporções corporais, relativamente às crianças nas quais foi baseada a referência antropométrica.

Trowbridge et al. ${ }^{1}$, estudando os efeitos da proporcionalidade corporal na composição corporal de crianças com baixa estatura, utilizaram isótopo estável de água para examinar a gordura e sua distribuição em 139 crianças. Encontraram que a espessura das pregas cutâneas tricipital e subescapular e a área de gordura do braço eram inferiores, mas, o total de água era mais elevado nessas crianças, quando comparado com os valores de referência. Desse modo, concluíram que o elevado valor de peso para estatura observado não deveria ser considerado obesidade, mas um reflexo da grande proporção ou hidratação da massa magra.

Considerando, portanto, que, além da relação peso para estatura, a avaliação do estado nutricional inclui também o conhecimento da composição corporal (massas magra e de gordura), tem-se buscado o desenvolvimento de indicadores antropométricos que melhor a reflitam. Teoricamente, tais indicadores não deveriam se correlacionar com a estatura, mas com a massa corporal e com medidas de gordura corporal, já que um indivíduo mais alto pode ter massa corporal maior decorrente de maior massa magra9.

O peso dividido pela estatura ao quadrado, chamado de Índice de Massa Corporal (IMC), é o mais utilizado para avaliação, tanto de sobrepeso quanto de obesidade, pois, além de ter sido validado em relação a medidas de densidade corporal, controla parcialmente 0 efeito da estatura ${ }^{10}$.

Entretanto, Garn et al. ${ }^{11}$ levantaram três limitações para o uso do IM C: a correlação com a estatura (que, apesar de baixa, ainda é significativa), com a massa magra (principalmente nos homens) e com a proporcionalidade corporal as quais, segundo os autores, fragilizam a utilização do IMC como indicador de gordura corporal.

Estudos brasileiros com populações adultas têm mostrado uma associação entre baixa estatura e sobrepeso, avaliado pelo IMC, a qual, talvez, seja resultado desse efeito da proporcionalidade corporal12,13.

Diante desses achados, deve-se levar em consideração a importância do ajustamento do IMC em relação à proporcionalidade corporal ${ }^{6}$. Norgan ${ }^{14}$ destaca que aborígines australianos, vivendo em condições adequadas, apresentam as maiores pernas, os menores valores de IC e os 
mais baixos de IMC do mundo. A contribuição das pernas longas para os baixos valores de IMC foi estimada em $2,0 \mathrm{~kg} / \mathrm{m}^{2}$. Outro estudo do mesmo autor mostrou que o ajuste do IMC em relação ao IC reduziu a porcentagem de indivíduos adultos classificados com baixo peso (IM C $<18,5 \mathrm{~kg} / \mathrm{m}^{2}$ ) de $30 \%$ para $7 \%$ e aumentou o diagnóstico de sobrepeso (IM C $\left.\geq 25,0 \mathrm{~kg} / \mathrm{m}^{2}\right) \mathrm{de}$ $8 \%$ para $15 \%{ }^{15}$.

Analogamente, em grupos com elevados valores de IC, como em indígenas sul-americanos, observou-se redução na prevalência de sobrepeso ao ajustar o IMC em função da proporcionalidade corporal. Mesmo em populações nas quais há pouca variação do IC, com a maioria dos indivíduos apresentando valores medianos, as diferenças na proporcionalidade corporal podem ser mais um fator a influenciar o IMC, somando-se aos efeitos das variáveis relativas à composição corporal6.

Entretanto, antes de questionar a validade do IMC na avaliação antropométrica de populações com comprometimentos na estatura e na proporcionalidade corporal, é importante considerar o comportamento da composição corporal nesses indivíduos. Para isso, é necessário verificar medidas de adiposidade, permitindo discriminar a composição da massa corporal encontrada. Entre essas medidas, estão as espessuras de pregas cutâneas que podem ser utilizadas em valores absolutos ou em equações de regressão para predição do percentual de gordura corporal $(\% \text { Gord })^{16}$.

Contudo, mesmo sabendo-se o \% Gord e, daí, a quantidade total de massa magra e massa adiposa, pode não ser possível uma avaliação adequada em termos de composição corporal. Indivíduos com diferentes estaturas podem ter massas e percentuais de gordura similares, ainda que estado nutricional diferenciado. 0 efeito da variação de estatura sobre a massa adiposa torna importante a utilização do Índice de Massa Corporal de Gordura (IMCG), calculado a partir da fração corporal de gordura ${ }^{10,17}$.
Velásquez-M eléndez et al. ${ }^{18}$, em estudo com brasileiras adultas, encontraram que o maior IC pode, potencialmente, ser fator de risco independente para 0 acúmulo de gordura corporal subcutânea e global. A partir disso, sugeriram que os fatores que potencialmente afetam o IC, como determinantes genéticos, condições ambientais e nutricionais, sejam estudados em sociedades em desenvolvimento como uma possível nova causa de aumento da obesidade em sociedades transicionais.

O presente estudo objetivou, portanto, investigar a distribuição dos valores de IC em uma população de adolescentes pós-menarca e verificar como as medidas e índices antropométricos variam em indivíduos com diferentes proporções corporais. Coloca-se em questão a aplicabilidade dos índices de peso relativo para estatura, em especial o IMC de acordo com a idade, utilizado no Brasil para avaliação antropométrica de adolescentes. Tal índice pode não ser tão adequado para avaliar populações com diferentes padrões ambientais e/ou genéticos que levem a diferentes perfis de composição, tamanho e proporções corporais ${ }^{1}$, gerando diagnósticos antropométricos inexatos.

Na população adolescente, esses diagnósticos inexatos podem ter repercussões ainda mais graves, considerando sua excessiva preocupação com o corpo e a conseqüente vulnerabilidade, sobretudo no sexo feminino, a comportamentos nocivos à saúde, visando atingir ideais estéticos de magreza.

\section{M É T O D O S}

Realizou-se um estudo transversal com 80 adolescentes do sexo feminino, estudantes de uma escola pública, no município de Alfenas, MG, durante o período de março a maio de 2005. 0 cálculo amostral baseou-se no estudo de Vieira et al. ${ }^{19}$, que encontrou significância estatística, ao nível de 5\%, utilizando amostra de 80 indivíduos, para verificação da influência da proporcionalidade corporal na avaliação pôndero-estatural dessa população específica. 
Utilizou-se formulário padronizado para entrevista, incluindo, além do nome e endereço completos, data de nascimento e idade da menarca. Subtraindo-se a idade da menarca da idade cronológica, foi determinada a idade ginecológica, em anos ${ }^{5}$.

Incluíram-se somente adolescentes com pelo menos 12 meses pós-menarca, objetivando maior probabilidade de terem ultrapassado 0 período mais intenso de mudanças físicas, relacionadas à puberdade 5 . A faixa etária considerada (10-20 anos) baseou-se na definição de adolescência da Organização Mundial da Saúde $(\mathrm{OMS})^{20}$. Esses procedimentos também foram adotados com o objetivo de homogeneizar a amostra em estudo.

Para as comparações entre as medidas e os índices antropométricos, as adolescentes foram distribuídas em dois grupos, com base na mediana do IC da população. O Grupo de Estudo (GE) correspondeu ao IC maior que a mediana (IC $>0,52-n=37$ ), com maior probabilidade de apresentarem comprometimentos na proporcionalidade corporal, e o Grupo Controle (GC) correspondeu ao IC menor ou igual à mediana $(I C \leq 0,52-n=43)$.

Para aferição da estatura, utilizou-se antropômetro portátil, constituído por plataforma metálica para posicionamento dos indivíduos e coluna de madeira desmontável contendo fita milimetrada - com comprimento de $213 \mathrm{~cm}$ - e cursor para leitura. 0 peso foi aferido utilizando-se balança eletrônica portátil com capacidade para $150 \mathrm{~kg}$ e subdivisão de $100 \mathrm{~g}$, adotando-se as técnicas propostas por Jellife ${ }^{21}$. A estatura tronco-cefálica (ETC) foi obtida por meio do mesmo equipamento utilizado para a aferição da estatura. 0 indivíduo foi medido sentado em um banco com altura previamente aferida, utilizando-se também o mesmo equipamento. A altura do banco foi, então, subtraída da estatura apresentada pelo indivíduo na posição sentada ${ }^{1,5}$. Subtraindo-se a ETC da estatura do indivíduo, foi obtida a medida do comprimento de pernas $(\mathrm{CP})^{8}$.
As pregas cutâneas foram aferidas utilizando-se adipômetro, com pressão constante de $10 \mathrm{~g} / \mathrm{mm}^{2}$ e precisão de $1 \mathrm{~mm}$, no lado direito do corpo ${ }^{22}$. A aferição das circunferências da cintura, quadril e abdome seguiu a metodologia proposta por Lohman et al. ${ }^{23}$, utilizando-se fita métrica inextensível.

Calculou-se o IMC por meio da relação entre o peso e a estatura ao quadrado $\left(\mathrm{P} / \mathrm{E}^{2}\right) .0$ Índice de Cormic foi obtido pela razão entre as medidas de ETC e estatura ${ }^{8}$.

Obteve-se $0 \%$ Gord por bioimpedância vertical (BIA ${ }^{24}$ e a massa de gordura corrigida pela estatura - IM CG: massa de gordura (kg)/estatura $^{2}(m)$ - foi calculada a partir dos valores de $\%$ Gord e peso ${ }^{17}$. 0 \% Gord, o IMCG e o somatório das pregas cutâneas foram considerados indicadores de adiposidade global. As circunferências da cintura, do abdome e do quadril, bem como as medidas de pregas cutâneas, foram utilizadas como indicadores de adiposidade localizada18,23.

O teste de Bartlett's foi utilizado para verificar a homogeneidade das distribuições amostrais e a possibilidade de aplicar estatística paramétrica ou não-paramétrica. Para comparação entre GE e GC, foram aplicados os testes do Qui-Quadrado, para análise de variáveis categóricas, e os testes " $t$ " de Student ou M ann-Whitney, para variáveis contínuas. Estabeleceu-se nível de significância de 5\% $(p<0,05)$ para todos os testes estatísticos. Para a armazenagem e análise dos dados foram utilizados os softwares Epi Info 6.04 e Stata 9.1.

O trabalho observou as normas da Resolução 196, do Conselho Nacional de Saúde, que regulamenta as pesquisas envolvendo seres humanos, tendo obtido aprovação pelo Comitê de Ética em Pesquisa com Seres Humanos da Universidade Federal de Alfenas.

\section{RES U LTA D O S}

Para verificar a homogeneidade dos grupos de adolescentes comparados, foi realizada a 
caracterização da população em relação às idades cronológica e ginecológica, tendo-se observado que não houve diferença significante entre os grupos em relação a essas variáveis (Tabela 1).

A distribuição de freqüência dos valores de IC encontrados na população de adolescentes estudada está apresentada na Figura 1. A média

Tabela 1. Idades cronológica e ginecológica, em anos, das adolescentes do grupo de estudo (GE) e do grupo controle (GC). Alfenas, MG, 2005.

\begin{tabular}{lrrrcc}
\hline \multirow{2}{*}{ Idade (anos) } & \multicolumn{2}{c}{ Cronológica } & & \multicolumn{2}{c}{ Ginecológica } \\
\cline { 2 - 3 } \cline { 5 - 6 } & $\mathrm{GE}(\mathrm{n}=37)$ & $\mathrm{GC}(\mathrm{n}=43)$ & & $\mathrm{GE}(\mathrm{n}=36)^{*}$ & $\mathrm{GC}(\mathrm{n}=42)^{*}$ \\
\hline M édia & 16,46 & 16,58 & & 4,36 & 4,19 \\
Desvio-padrão & 1,55 & 1,25 & & 1,80 & 1,50 \\
Mediana & 17,00 & 17,00 & & 4,00 & 4,00 \\
\hline
\end{tabular}

* Uma adolescente não soube informar a idade da menarca. Teste " $\mathrm{t}$ " de Student: Idade cronológica $p=0,69$; Idade ginecológica $p=0,66$. e o desvio-padrão do IC foram de 0,52 e 0,013, respectivamente. 0 teste de Shapiro-Wilk não mostrou significância estatística $(p=0,77)$, indicando que o IC apresenta uma distribuição normal nesta população.

A Tabela 2 apresenta os valores médios e medianos de peso, estatura, IMC, ETC e CP. Os resultados mostraram que os valores de peso e estatura não diferiram entre os grupos estudados. Por outro lado, as variáveis ETC e IMC apresentaram-se significantemente superiores no grupo de estudo $(p<0,001 ; p<0,01)$. Os valores do comprimento de pernas, analogamente, foram superiores no grupo controle $(p<0,001)$.

A análise da comparação entre GE e GC, considerando a variável IMC categorizada de acordo com a mediana da população (Figura 2),

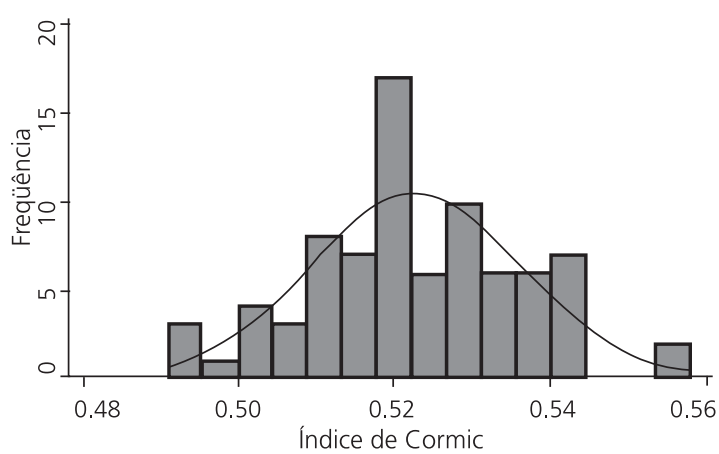

Figura 1. Distribuição de freqüência do Índice de Cormic de adolescentes do sexo feminino $(n=80)$. Alfenas, MG, 2005.

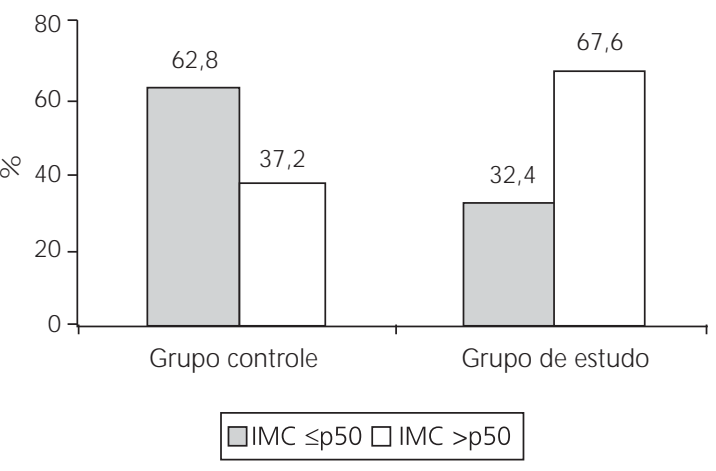

Figura 2. Associação entre Índice de Cormic e Índice de Massa Corporal (IMC), em adolescentes pós-menarca $(\mathrm{n}=80)$. Alfenas, MG, 2005. Teste do Qui-quadrado $p<0,01$.

Tabela 2. Peso (kg), Estatura (m), Índice de Massa Corporal (IMC) $\left(\mathrm{kg} / \mathrm{m}^{2}\right)$, Estatura tronco-cefálica (ETC) (m) e Comprimento de pernas (CP) (m), das adolescentes do grupo de estudo (GE) e do grupo controle (GC). Alfenas, MG, 2005.

\begin{tabular}{|c|c|c|c|c|c|c|c|}
\hline \multirow{2}{*}{ Variáveis antropométricas } & \multicolumn{3}{|c|}{ GE $(n=37)$} & \multicolumn{3}{|c|}{ GC $(n=43)$} & \multirow{2}{*}{$\mathrm{p}$} \\
\hline & Média & DP & Mediana & Média & $\mathrm{DP}$ & Mediana & \\
\hline Peso & 56,91 & 9,47 & 58,00 & 53,47 & 7,27 & 51,20 & 0,069 \\
\hline Estatura & 1,60 & 0,06 & 1,59 & 1,60 & 0,05 & 1,60 & 0,23 \\
\hline IMC & 22,24 & 3,67 & 21,30 & 21,00 & 2,50 & 20,00 & $<0,01$ \\
\hline ETC & 0,85 & 0,29 & 0,86 & 0,83 & 0,28 & 0,83 & $<0,001$ \\
\hline$C P$ & 0,75 & 0,05 & 0,74 & 0,80 & 0,03 & 0,78 & $<0,001$ \\
\hline
\end{tabular}

Teste " $\mathrm{t}$ " de Student, exceto para IMC, em que se aplicou teste de Mann-Whitney; DP: desvio-padrão. 
Tabela 3. Medidas de adiposidade global e localizada das adolescentes do grupo de estudo (GE) e do grupo controle (GC). Alfenas, MG, 2005.

\begin{tabular}{|c|c|c|c|c|c|c|c|}
\hline \multirow{2}{*}{ Variáveis antropométricas } & \multicolumn{3}{|c|}{ GE $(n=37)$} & \multicolumn{3}{|c|}{$\mathrm{GC}\left(\mathrm{n}=41^{*}\right)$} & \multirow{2}{*}{$p$} \\
\hline & Média & DP & Mediana & M édia & $\mathrm{DP}$ & Mediana & \\
\hline$\%$ Gord & 30,01 & 3,29 & 30,50 & 30,22 & 4,92 & 30,00 & 0,87 \\
\hline S4P (mm) & 76,64 & 23,62 & 74,00 & 72,98 & 6,38 & 68,00 & 0,46 \\
\hline PCT (mm) & 21,42 & 6,38 & 21,00 & 20,37 & 5,53 & 19,00 & 0,44 \\
\hline PCB (mm) & 14,28 & 5,75 & 14,00 & 13,39 & 4,38 & 13,00 & 0,46 \\
\hline PCSE (mm) & 17,28 & 6,07 & 16,00 & 16,07 & 5,55 & 14,00 & 0,36 \\
\hline PCSI (mm) & 23,65 & 7,70 & 24,00 & 23,15 & 6,70 & 22,00 & 0,76 \\
\hline $\mathrm{CQ}(\mathrm{cm})$ & 96,35 & 7,27 & 96,00 & 93,35 & 6,47 & 92,50 & 0,06 \\
\hline $\mathrm{CA}(\mathrm{cm})$ & 80,91 & 8,43 & 80,50 & 78,94 & 7,04 & 77,00 & 0,26 \\
\hline $\mathrm{CC}(\mathrm{cm})$ & 69,65 & 7,60 & 68,50 & 67,92 & 8,29 & 67,50 & 0,34 \\
\hline
\end{tabular}

Teste "t" de Student

* Duas adolescentes do GC não concordaram em realizar essas medidas; \% Gord: Porcentagem de gordura corporal; S4P: Somatório das 4 pregas cutâneas; PCT: Prega Cutânea Tricipital; PCB: Prega Cutânea Bicipital; PCSE: Prega Cutânea Subescapular; PCSI: Prega Cutânea Suprailíaca; CQ: Circunferência do Quadril; CA: Circunferência do Abdome; CC: Circunferência da Cintura; DP: Desvio-padrão.

mostrou que proporção significantemente superior $(67,6 \%)$ de adolescentes do GE apresentou IM C acima da mediana da população, quando comparadas às adolescentes do GC (37,2\%).

Em relação ao \% Gord, não foi observada diferença estatística entre GE e GC (Tabela 3). Essa semelhança entre os grupos permaneceu mesmo após a correção da massa de gordura pela estatura, por meio do IMCG.

Os valores de pregas cutâneas e circunferências (Tabela 3) também não diferiram, estatisticamente, entre os grupos.

\section{I S C U S S Ã O}

Com o objetivo de homogeneizar a população em estudo, foram selecionadas estudantes de escolas públicas que, teoricamente, pertencem a nível socioeconômico mais baixo, tendo, assim, maior probabilidade de terem sido expostas a riscos nutricionais ao longo da vida ${ }^{5}$.

A semelhança entre os grupos estudados em relação às idades cronológica, ginecológica e da menarca é conveniente para que os resultados das demais comparações entre os dois grupos sejam independentes de possíveis influências do estágio de maturação sexual das adolescentes ${ }^{5}$. Isso porque os eventos da puberdade relacionam- -se ao estado nutricional, especialmente ao perfil de composição corporal e, portanto, esse aspecto constitui um potencial fator de confusão ${ }^{25}$.

Os resultados relativos à idade ginecológica indicam que essas adolescentes já ultrapassaram o período mais intenso de modificações corporais relacionadas à puberdade, sendo os grupos em estudo equiparáveis em relação ao estágio de desenvolvimento ${ }^{5}$.

A mediana do IC encontrada neste estudo correspondeu a 0,52 , valor inferior ao encontrado em estudo realizado na cidade de Kitakyueh, Japão, com 318 adolescentes do sexo feminino, de aproximadamente 17 anos. Observou-se que - IC dessas adolescentes japonesas foi de aproximadamente 0,54 , valor próximo aos encontrados em chineses, esquimós, índios americanos e hokkaido-ainu²6.

Valores maiores de IC devem-se à maior proporção tronco-cefálica, ou seja, troncosmaiores em relação às pernas, ou pernas mais curtas, condição possivelmente resultante de comprometimentos nutricionais ao longo da vida ${ }^{4,5}$.

Os resultados encontrados em relação às variáveis estatura tronco-cefálica (ETC), comprimento de pernas (CP) e estatura, permitem inferir que as adolescentes do GE, com maior IC, apresentaram esse comprometimento na proporcionalidade corporal em virtude do menor $\mathrm{CP}$, em 
relação ao GC, já que suas estaturas foram semelhantes. Considerando que a medida da ETC foi significantemente maior nessas adolescentes do $G E$, provavelmente, elas seriam mais altas que as pertencentes ao $\mathrm{GC}$, não fosse o comprometimento na proporcionalidade, expresso no $\mathrm{CP}$ reduzido.

Analogamente, pode-se dizer que as adolescentes do GC seriam mais baixas por uma possível herança genética e, portanto, sem comprometimento na proporcionalidade corporal ${ }^{4}$.

O comprimento de pernas tem sido considerado um indicador mais sensível da exposição dos indivíduos a circunstâncias socioeconômicas, dietéticas e ambientais desfavoráveis no perío do da infância ${ }^{27,28}$. Para Wadsworth et al. ${ }^{27}, 0$ comprimento das pernas é particularmente sensível aos fatores ambientais e à dieta até os 5 anos, pois esse é o perío do de crescimento mais rápido desse segmento corporal. Gunnel et al. ${ }^{28}$ apontam ainda para a possibilidade de riscos de doenças na idade adulta em indivíduos com pernas mais curtas.

As adolescentes do $G E$, que possuíam estaturas semelhantes às do $G C$, apresentaram maiores valores de peso, determinando maior relação peso para estatura nesse grupo.

Post et al. ${ }^{8}$ avaliaram índices antropométricos, inclusive de proporcionalidade corporal, de crianças com e sem déficit estatural, buscando explicações para a baixa prevalência de déficit de peso para estatura entre crianças com déficit estatural. Concluíram que essa baixa prevalência não resultou de excesso de tecido adiposo ou de massa muscular, mas do crescimento isolado do abdome e, principalmente, do maior IC e menor proporção de pernas entre crianças com déficit estatural. O CP das crianças com déficit estatural foi a medida que mais apresentou diferença em relação às crianças norte-americanas, sendo menos marcantes as diferenças na ETC. Para os autores, esse fator poderia ser atribuído à taxa de maturação lenta das crianças pobres e seria uma possível explicação para a baixa prevalência de déficit de peso para estatura na presença de elevada freqüência de déficit estatural. Isso porque cabeça e tronco, possivelmente, sejam os segmentos que contribuem mais significativamente para o peso corporal.

$\mathrm{O}$ estudo de Vieira et al. ${ }^{19}$ verificou a influência do IC na avaliação pôndero-estatural de adolescentes pós-menarca, em Viçosa, MG. Observou-se que proporção significantemente superior $(64,3 \%)$ de adolescentes no 3 o tercil de IC apresentou valores de IM C acima da mediana da população, em relação às adolescentes no 1 응 tercil $(34,6 \%)$. Para as autoras, tais resultados indicam que altos valores de IC podem, de fato, estar associados a elevado IMC, questionando-se a utilização de índices de peso relativo na avaliação de populações com comprometimentos estaturais e na proporcionalidade corporal.

Velásquez-M eléndez et al. ${ }^{18}$ investigaram se elevados valores de IC estavam independentemente relacionados ao IM C, em 669 mulheres adultas de Belo Horizonte, MG. 0 ponto de corte adotado para elevado IC foi o valor médio mais 1 desvio-padrão. Os resultados das análises multivariadas, com ajuste para possíveis variáveis de confusão - como idade, escolaridade, estado civil, idade da menarca, paridade e obesidade dos pais - mostraram que as mulheres com elevado IC apresentaram 2,46 vezes mais chance de apresentar IM C $>30 \mathrm{~kg} / \mathrm{m}^{2}$.

Baugust \& Walley ${ }^{29}$ argumentam que, apesar de o IMC ter sido adotado pela OM S como uma medida internacional de obesidade, existe ainda uma deficiência de base teórica e experiências empíricas sugerem que seu uso não é válido para todas as populações, podendo ser comprometido por diferenças étnicas, culturais e ambientais.

Deve-se, portanto, levar em consideração a importância do ajustamento do IM C em relação à proporcionalidade corporal, conforme mostraram Norgan \& Jones ${ }^{6}$. Com a correção do IMC em relação ao IC, observou-se uma mudança no IM C entre uma e duas unidades em $10 \%$ dos homens e $33 \%$ das mulheres, e de duas unidades em $1 \%$ dos homens e $5 \%$ das mulheres. Segundo os autores, a diferença de uma unidade no IMC, ou 
seja, $1 \mathrm{~kg} / \mathrm{m}^{2}$, é importante, pois significa uma variação de peso de $2,5-3,0 \mathrm{~kg}$, o que equivale ao ganho de peso em um período de sete anos, durante a fase adulta.

Indivíduos que apresentam maior IC poderiam apresentar também maior \% Gord, visto que, na região abdominal, diferentemente dos membros inferiores, há tecido adiposo visceral e o subcutâneo ${ }^{30,31}$. De forma análoga, as pernas mais curtas, proporcionalmente, poderiam determinar menor percentual de massa magra e, conseqüentemente, maior \% Gord.

0 estudo de Bénèfice et al. ${ }^{25}$ aponta nessa direção, visto que adolescentes com baixa estatura diferiam significantemente quanto aos valores das pregas cutâneas bicipital e subescapular, sugerindo maior depósito de gordura subcutânea na parte superior do corpo.

Vieira ${ }^{5}$, diferentemente, não encontrou no grupo com baixa estatura (e maior IC) quantidade superior de gordura subcutânea no tronco, avaliada por pregas cutâneas, em relação ao grupo com estatura normal. Além disso, a baixa estatura também não foi associada a maior circunferência da cintura (CC), também utilizada no estudo para avaliar a topografia da gordura corporal.

0 estudo de Velásquez-Meléndez et al. ${ }^{18}$ também investigou se elevados valores de IC estavam independentemente relacionados à adiposidade, global e localizada. Os resultados das análises multivariadas revelaram que variáveis como $0 \%$ Gord, estimado por bioimpedância elétrica, e o somatório das pregas cutâneas apresentaram valores médios maiores no grupo de mulheres com alto IC. Essas mulheres apresentaram 2,07 e 2,11 vezes mais chance de apresentar \% Gord acima de $30 \%$ e somatório das pregas cutâneas no 30 tercil, respectivamente. Além disso, as medidas das circunferências da cintura e do quadril também eram significantemente maiores, levando os autores a concluírem que o maior IC pode ser um fator de risco independente para maior adiposidade nessas regiões.

Tais asso ciações não foram encontradas no presente estudo, apesar de terem sido observadas diferenças numéricas entre os grupos. A amostra de 80 adolescentes, bastante inferior à considerada no estudo de Velásquez-M eléndez et al. ${ }^{18}$ ( $n=669$ ), não permitiu a adoção do mesmo critério utilizado por esses autores para classificar o IC como elevado, qual seja, a média mais 1 desvio-padrão. Tal critério poderia proporcionar maior contraste entre os grupos aqui estudados e, conseqüentemente, maior possibilidade de diferenças estatisticamente significantes para as variáveis relacionadas à gordura corporal.

\section{O N C L U S Ã O}

A maioria dos estudos centra-se apenas na avaliação da estatura dos indivíduos e não no comportamento de seus componentes. Esses componentes - estatura tronco-cefálica e comprimento de pernas, indicadores da proporcionalidade corporal - podem diferir ao longo do crescimento. 0 fato de não terem sido encontradas, no presente estudo, diferenças estaturais entre as adolescentes com e sem comprometimento na proporcionalidade corporal é relevante, sobretudo no que concerne à definição de indicadores antropométricos do estado nutricional.

Verificou-se a influência da proporcionalidade corporal na relação pôndero-estatural das adolescentes pós-menarca, indicando que altos valores de IC podem estar associados a elevado IMC. Entretanto, tal influência não foi verificada na avaliação da composição corporal e da topografia da gordura corporal dessas adolescentes. A partir desses resultados, questiona-se a utilização do IMC como indicador antropométrico em adolescentes, principalmente nos serviços de saúde de países em desenvolvimento, como o Brasil, em que parcelas significativas da população ainda sofrem privações nutricionais que as tornam suscetíveis a comprometimentos na proporcionalidade corporal. Esses indivíduos podem apresentar seu diagnóstico antropométrico "mascarado", apresentando maior IM C e não necessariamente mais adiposidade global ou localizada. 


\section{A G RA DECIM ENTO}

À Profa. Sílvia Eloiza Priore, do Departamento de Nutrição e Saúde da Universidade Federal de Viçosa, pela leitura cuidadosa deste manuscrito, contribuindo com correções e valiosas sugestões.

\section{REFERÊ N C I A S}

1. Trowbridge FL, Marks JS, Romano GL, Madrid S, Boutton TW, Klein PD. Body composition of Peruvian childrem with short stature and high weight-for-height. II-Implications for the interpretation for weight-for height as an indicator of nutritional status. Am J Clin Nutr. 1987; 46(3):411-8.

2. Frongillo EA. Symposium: causes and etiology of stunting. J Nutr. 1999; 129(Suppl. 2):529S-30S.

3. Norgan NG. Population differences in body composition in relation to the body mass index. Eur J Clin Nutr. 1994; 48(Suppl. 3):S10-25.

4. Henneberg M, Harrison GA, Brush G. The small child: anthropometric and physical performance characteristics of short-for-age children growing in good and in poor socio-economic conditions. Eur J Nutr. 1998; 52(4):286-91.

5. Vieira VCR. Baixa estatura em adolescentes pós-menarca: abordagem nutricional retrospectiva e atual [dissertação]. Viçosa: Universidade Federal de Viçosa; 2003.

6. Norgan NG, Jones PRM. The effect of standardizing the body mass index for relative sitting height. Int J Obes. 1995; 19(3):206-8.

7. Popkin BM, Richards MK, M onteiro CA. Stunting is associated with overweight in children of four nations that are undergoing the nutrition transition. J Nutr. 1996; 126(12):3009-16.

8. Post CLA, Victora, CG, BarrosAJD. Baixa prevalência de déficit de peso para estatura: comparação de crianças brasileiras com e sem déficit estatural. Rev Saúde Pública. 1999; 33(6):575-85.

9. Anjos LA. Índice de massa corporal como indicador do estado nutricional de adultos: revisão de literatura. Rev Saúde Pública. 1992; 26(6):431-6.

10. Bellizzi MC, Dietz WH. Workshop on childhood obesity: summary of the discussion. Am J Clin Nutr. 1999; 70(1):173S-5S.

11. Garn SM, Leonard WR, Hawthorne VM. Three limitations of the body mass index. Am J Clin Nutr. 1986; 44(6):996-7.

12. Sichieri R, Siqueira KS, Moura AS. Obesity and abdominal fatness associated with undernutrition early in life in a survey in Rio de Janeiro. Int J Obes. 2000; 24(5):614-8.

13. Velásquez-M eléndez $G, M$ artins IS, Cervato $A M$, Fornés NS, Maruci M FN, Coelho LT. Relationship between stature, overweight and central obesity in the adult population in São Paulo, Brazil. Int J Obes. 1999; 23(6):639-44.

14. Norgan NG. On the interpretation of low body mass indices: Australian Aborigines. Am J Clin Nutr. 1994; 94(2):229-37.

15. Norgan NG. Body mass index and nutritional status - the effect of adjusting the body mass index for relative sitting height on estimates of the prevalence of chronic energy deficiency, overweight and obesity. Asia Pacific J Clin Nutr. 1995; 4(1): 137-9.

16. Sigulem DM, Devincenzi UM, Lessa AC. Diagnóstico do estado nutricional da criança e do adolescente. J Pediatr. 2000; 76(Suppl 3): S275-84.

17. Vanitallie TB, Yang MU, Heymsfield SB, Funk RC, Boileau RA. Height-normalized indices of the body's fat-free mass and fat mass: potentially useful indicator of nutritional status. Am J Clin Nut. 1990; 52(6):953-9.

18. Velásquez-M eléndez G, Silveira EP, Souza PA, Kac G. Relationship between sitting-height-to-stature ratio and adiposity in brazilian women. Am J Hum Biol. 2005; 17(5):646-53.

19. Vieira VCR, Bronhara B, Priore SE, Franceschini SCC. Influência da proporcionalidade corporal na avaliação pôndero-estatural em adolescentes pós-menarca. In: Anais do I Congresso M ineiro de Alimentação e Nutrição, 2005; Ouro Preto, Brasil. Ouro Preto: UFOP; 2005. CD-ROM.

20. Organización Mundial de La Salud. Necesidades de salud de los adolescentes. Ginebra: OM S; 1977. Série de Informes Técnicos, n.724.

21. Jelliffe DBI. Evaluación del estado de nutrición de la comunidad. Ginebra: OMS; 1968.

22. Sangi $H$, Mueller WH. Which M easure of Body Fat Distribution is Best for Epidemiologic Research among Adolescents? Am J Epidemiol. 1991; 133(9):870-83.

23. Lohman T, Roche AF, Martorell R. Anthropometric Standardization Reference Manual. Champaing: Human Kinetics Books; 1988.

24. Sampei MA, Novo NF, Juliano Y, Sigulem DM. Comparison of the body mass index to other methods of body fat evaluation in ethnic Japanese and Caucasian adolescent girls. Int J Obes Relat Metab Disord. 2001; 25(3):400-8.

25. Bénèfice $E$, Garnier $D$, Simondon KB, Malina RM . Relationship between stunting in infancy and 
growth and fat distribution during adolescence in Senegalese girls. Eur J Clin Nutr. 2001; 55(1): 50-8.

26. Hojo T, Nakashima T, Hirao T. Stature, sitting height and relative sitting height of female northern Kyushuites. J UOEH. 1984; 6(4):355-7.

27. Wadsworth MEJ, Hardy RJ, Paul AA, Marshal SF, Cole TJ. Leg and trunk length at 43 years in relation to childhood health, diet and family circumstances; evidence from the 1946 national birth cohort. Int J Epidemiol. 2002; 31(2):383-90.

28. Gunnel DJ, Smith GD, Frankel S, Nanchahal K, Bradon FEM, Pemberton J, et al. Childhood leg length and adult mortality: follow up of the Carnegie (boyd orr) survey of diet and health in pre-war Britain. J Epidemiol Community Health. 1998; 52(3):142-52.
29. Baugust A, Walley T. An alternative to body mass index for standardizing body weight for stature. Q J Med. 2000; 93(9):589-96.

30. Caprio S, Hyman LD, McCarthy S, Lange R, Bronson $M$, Tamborlane WV . Fat distribution and cardiovascular risk factors in obese adolescent girls: importance of the intraabdominal fat depot. Am J Clin Nutr. 1996; 64(1):12-7.

31. Han TS, McNelly G, Campbell D. The relation between women's birth weight and their current intra-abdominal fat-mass. Proc Nutr Soc. 1995; 54(3):182A.

Recebido em: 16/12/2005

Versão final reapresentada em: 24/11/2006 Aprovado em: 6/12/2006 
O arquivo disponível sofreu correções conforme ERRATA publicada no Volume 20 Número 4 da revista. 